\title{
Enterotoxigenic Escherichia coli infection alters intestinal immunity in mice
}

\author{
XIANGWU YANG ${ }^{1}$, ZHIMING XIAO $^{2}$, FEN LIU ${ }^{2}$, SHUAI CHEN ${ }^{3}$, WULIANG TANG ${ }^{2}$, \\ DECAI ZHANG ${ }^{2}$ and SHAOJUN LIU ${ }^{2}$
}

\begin{abstract}
Departments of ${ }^{1}$ Gastrointestinal Surgery and ${ }^{2}$ Digestion Medicine, The Third Affiliated Hospital of Xiang Ya School of Medicine, Central South University, Changsha, Hunan 410078; ${ }^{3}$ Key Laboratory of Agro-ecological Processes in Subtropical Region, Institute of Subtropical Agriculture,

Chinese Academy of Sciences, Changsha, Hunan 410125, P.R. China
\end{abstract}

Received March 23, 2015; Accepted April 7, 2016

DOI: $10.3892 / \mathrm{mmr} .2016 .5302$

\begin{abstract}
Enterotoxigenic Escherichia coli (ETEC) is a major cause of diarrhea in humans and piglets. However, research regarding alterations of intestinal immunity following ETEC infection remains limited and the results controversial. The present study investigated the effects of ETEC on the expression levels of pro-inflammatory cytokines and innate immune regulators from plasma cells, goblet cells and Paneth cells, and the activation of toll-like receptor 4-nuclear factor (NF) $-\kappa \mathrm{B}$ and mitogen-activated protein kinase (MAPK) pathways using reverse transcription-quantitative polymerase chain reaction and western blot analysis, in a mouse model infected with a porcine isolated ETEC strain. ETEC infection significantly reduced the expression of pro-inflammatory cytokines in the mouse jejunum $(\mathrm{P}<0.05)$. Additionally, ETEC infection significantly affected the expression of immune regulators of plasma cells, goblet cells and Paneth cells in the mouse intestine $(\mathrm{P}<0.05)$. ETEC influenced the intestinal immunity via the NF- $\kappa \mathrm{B}$ and MAPK signaling pathways. In conclusion, ETEC colonization affects intestinal immunity as observed in a mouse model. This study provides a greater understanding of the pathogenesis of ETEC infection in animals and humans.
\end{abstract}

\section{Introduction}

Enterotoxigenic Escherichia coli (ETEC) is one of the leading causes of bacterial diarrhea in humans and pigs. ETEC colonizes the intestine by fimbrial adhesins

Correspondence to: Professor Shaojun Liu, Department of Digestion Medicine, The Third Affiliated Hospital of Xiang Ya School of Medicine, Central South University, 138 Tongzhipo Road, Changsha, Hunan 410078, P.R. China

E-mail: cslsj169@126.com

Key words: enterotoxigenic Escherichia coli, NF-кB, paneth cells, mouse
(K88/F4, K99/F5, 987P/F6, F41/F7 and F18), and then produces enterotoxins, such as heat labile (LT), heat-stable (STa and STb), and entero-aggregative Escherichia coli heat-stable enterotoxin-1, which leads to excessive loss of fluids and electrolytes resulting in profuse diarrhea, frequently without histological lesions $(1,2)$. The alterations of intestinal function following ETEC colonization are widely investigated, including autophagy (3), immune responses (4-7), nutrient absorption (8), barrier integrity (9-11) and apoptosis $(12,13)$.

However, investigations regarding the intestinal immune response have remained controversial. Finamore et al (4) identified that ETEC (K88) infection activates the toll-like receptor 4 (TLR4)-nuclear factor $(\mathrm{NF})-\kappa \mathrm{B}$ signaling pathway by increasing the expression levels of TLR4 and myeloid differentiation primary response 88 . The phosphorylation of the conserved helix-loop-helix ubiquitous kinase [CHUK; also termed inhibitor of $\kappa \mathrm{B}(\mathrm{I} \kappa \mathrm{B}) \mathrm{kinase}(\mathrm{IKK}) \alpha]$, IKK $\beta$, $\mathrm{I} \kappa \mathrm{B} \alpha$ ) and $\mathrm{NF}-\kappa \mathrm{B}$ subunit $\mathrm{p} 65$, resulted in the overproduction of inflammatory cytokines interleukin (IL)-8 and IL-1 $\beta$ in Caco-2/TC7 cells and pig explants (5 week-old crossbreed Pietrain/Duroc/Large-White piglets) (4). McLamb et al (6) also reported thatETEC (F18) infection significantly promoted intestinal immune responses, identified elevation in IL-6 and -8 expression, neutrophil recruitment and mast cell activation in weaned pigs. However, Wang and Hardwidge (7) identified that ETEC blocks NF- $\mathrm{NB}$ signaling, which is commonly induced by tumor necrosis factor (TNF), IL-1 $\beta$, and flagellin by secreting a heat-stable, proteinaceous factor. A previous study identified that ETEC supernatant significantly blocks the degradation of the NF- $\kappa \mathrm{B}$ inhibitor $\mathrm{I} \kappa \mathrm{B} \alpha$ by preventing $\mathrm{I} \kappa \mathrm{B} \alpha$ polyubiquitination, without affecting $\mathrm{I} \kappa \mathrm{B} \alpha$ phosphorylation (7). A previous study indicated that ETEC infection inhibits the intestinal immune responses due to the fact that $\mathrm{NF}-\kappa \mathrm{B}$ is the principal pathway associated with the induction of host pro-inflammatory responses following infection $(14,15)$.

Thus, the present study was conducted to investigate the function of ETEC infection on intestinal immunity in a mouse model infected with a porcine isolated ETEC strain. 


\section{Materials and methods}

Bacterial strains and antibodies. The current study used ETEC298 (serotype O107; oqxAB; F18; STa, STb, SLT-IIe), which was a gift from Dr Wenkai Ren (Chinese Academy of Sciences, Changsha, China). It was isolated from piglets with diarrhea. ETEC298 was cultured in Luria-Bertani broth and on agar at $37^{\circ} \mathrm{C}$, as previously described $(3,16)$. Antibodies against mitogen-activated protein kinase 8 [MAPK8; also termed c-Jun N-terminal kinase (JNK)] (1:200; sc-571), phosphorylated-Jnk (1:200; sc-12882) and TLR4 (1:200; sc-10741), proliferating cell nuclear antigen (PCNA; 1:200; sc-56) and actin (1:200; SC-1616) were purchased from Santa Cruz Biotechnology, Inc. (Dallas, TX, USA). Antibodies against MAPK3 [also termed extracellular signal-related kinase 1/2 (ERK1/2)] (1:500; CST 4695), phosphorylated-ERK1/2 (1:500; CST 4370), p38 (1:500; CST 8690), p-p38 (1:500; CST 4511) and p65 (1:500; CST 6956) were purchased from Cell Signaling Technology, Inc. (Danvers, MA, USA).

Animal model. The current study was performed according to the guidelines of the Laboratory Animal Ethical Commission of the Institute of Subtropical Agriculture, Chinese Academy of Sciences (Changsha, China). The ETEC infection model was established according to the method described by Allen et al (17). Female ICR mice were purchased from Shanghai Laboratory Animal Center (Shanghai, China). The mice were housed in a pathogen-free mouse colony at $20-30^{\circ} \mathrm{C}, 45-60 \%$ humidity with a 12 -h light/dark cycle. At six weeks of age, mice (24-26 g, n=42 for ETEC infection; $\mathrm{n}=15$ for control) were randomly assigned into either the ETEC infection or control groups and inoculated by oral gavage with either $1 \times 10^{9}$ colony forming units ETEC298 or with sterile phosphate-buffered saline (PBS, Hunan World Well-being Bio-tech Co., Ltd., Hunan, China). Mice were sacrificed by cervical dislocation after $24 \mathrm{~h}$, and the jejunum and ileum was collected. Segments $(2 \mathrm{~cm})$ of the jejunum and ileum (middle part) were dissected after washing the intestinal contents with PBS. Tissue samples were shock cooled with liquid nitrogen. After shock freezing the samples were kept at $-80^{\circ} \mathrm{C}$ until further processing. The mice were used as a model to investigate the alterations of intestinal immunity following ETEC infection due to the fact that previous studies demonstrated that human or porcine isolated ETEC affects the intestinal functions of mice, although no diarrhea was observed subsequent to ETEC inoculation $(17,18)$. The dosage of ETEC for infection, and the time points for tissue collection were based on previous studies $(17,18)$. As the jejunum is the main target for ETEC in the host (18), thus, the current study also focused on the alterations of intestinal immunity in mouse jejunum.

Morphological analyses. For light microscopic observation (DM6M, Leica Microsystems, Wetzlar, Germany), jejunum tissues were fixed with 10\% formalin (Hunan World Well-being Bio-tech Co., Ltd.) and PBS at $4^{\circ} \mathrm{C}$, dehydrated in a graded series of ethanol, then embedded in paraffin wax (Hunan World Well-being Bio-tech Co., Ltd.). Tissue sections (5 $\mu \mathrm{m}$, cut by SYD-S2020; YuDe, Shengyang, China) were mounted on slides, dewaxed (in xylene, Sangon Biotech,
Co., Ltd., Shanghai, China), hydrated and then stained with hematoxylin \& eosin (Hunan World Well-being Bio-tech Co., Ltd.).

Immunoblotting. Equal amounts of proteins $(100 \mu \mathrm{g})$ obtained from cytoplasmic or nuclear fractions were separated by sodium dodecyl sulfate-polyacrylimide gel electrophoresis, transferred to polyvinylidene difluoride membranes (EMD Millipore, Billerica, MA, USA), and blocked with 5\% non-fat milk in Tris-buffered saline with Tween-20 (20 mM Tris, pH 7.5, $150 \mathrm{mM} \mathrm{NaCl}$ and $0.1 \%$ Tween-20 (Sangon Biotech, Co., Ltd.) for $3 \mathrm{~h}$. Membranes were incubated with primary antibodies overnight at $4^{\circ} \mathrm{C}$ and then with horseradish peroxidase conjugated-goat anti-rabbit $\operatorname{IgG}(1: 5,000$; cat. no. sc-2030; Santa Cruz Biotechnology, Inc. for $1 \mathrm{~h}$ at room temperature prior to development and analysis using 3.2 Alpha Imager 2200 software (Alpha Innotech Corporation, San Leandro, CA, USA). The resultant signals were digitally quantified and the data was normalized to PCNA or actin abundance. PCNA or actin was used as an internal loading control for nuclear and cytoplasmic protein fractions, respectively.

Reverse transcription-quantitative polymerase chain reaction $(R T-q P C R)$. Total RNA was isolated from liquid nitrogen frozen jejunum or ileum samples with TRIzol regent (Invitrogen; Thermo Fisher Scientific, Inc., Waltham, MA, USA) and then treated with DNase I (Invitrogen; Thermo Fisher Scientific, Inc.) according to the manufacturer's instructions. Total RNA $(1 \mu \mathrm{g})$ was reverse-transcribed to cDNA using the iScript cDNA synthesis kit (Bio-Rad, Hercules, CA, USA). Expression of target genes was assayed by RT-qPCR using SYBR Green mix (Takara Biotechnology (Dalian) Co., Ltd., Dalian, China). The primers used are as described in previous studies $(15,19)$. $\beta$-actin was used as an internal control to normalize target gene transcript levels. RT-qPCR was performed according to a previous study (20). Briefly, $1.0 \mu \mathrm{l}$ cDNA template was added to a total volume of $10 \mu \mathrm{l}$ containing $5 \mu \mathrm{l}$ SYBR Green mix (Takara Biotechnology (Dalian) Co., Ltd.), $0.2 \mu 1$ Rox

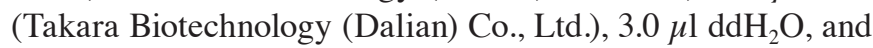
$0.4 \mu \mathrm{l}$ each of forward and reverse primers (Sangon Biotech, Co., Ltd.). The following cycling protocol was used with an ABI7900 thermocycler (Applied Biosysterms, Thermo Fisher Scientific, Inc.): i) Pre-denaturation $\left(10 \mathrm{sec}\right.$ at $\left.95^{\circ} \mathrm{C}\right)$; ii) amplification and quantification $\left(40\right.$ cycles of $5 \mathrm{sec}$ at $95^{\circ} \mathrm{C}$ and $20 \mathrm{sec}$ at $\left.60^{\circ} \mathrm{C}\right)$; iii) melting $\left(60-99^{\circ} \mathrm{C}\right.$ with a heating rate of $0.1^{\circ} \mathrm{C} / \mathrm{sec}$ and fluorescence measurement). Relative expression was normalized and expressed as a ratio to the expression in the control group.

Statistical analysis. Data are expressed as the mean \pm standard error. All statistical analyses were performed using SPSS software (version 16.0; SPSS, Inc., Chicago, IL, USA). Data were analyzed using Student's t-test and $\mathrm{P}<0.05$ was considered to indicate a statistically significant difference.

\section{Results}

Clinical results. For the mice, there was a mortality rate of 11 out of 42 (26\%) within $24 \mathrm{~h}$ of infection with ETEC298 strain, whereas there was a mortality rate of 0 out of 42 following 
A
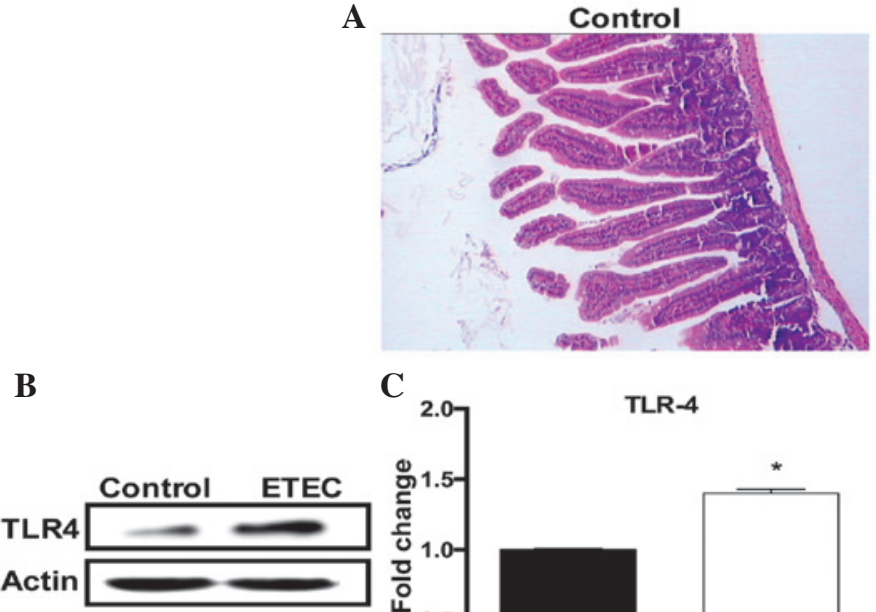

C

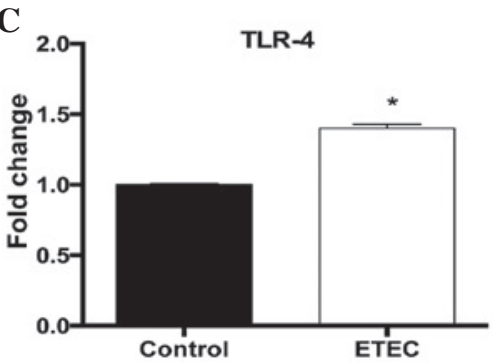

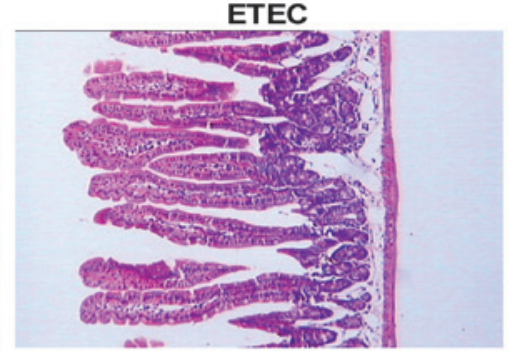

D

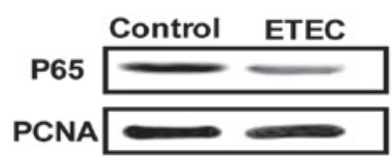

$\mathbf{E}$

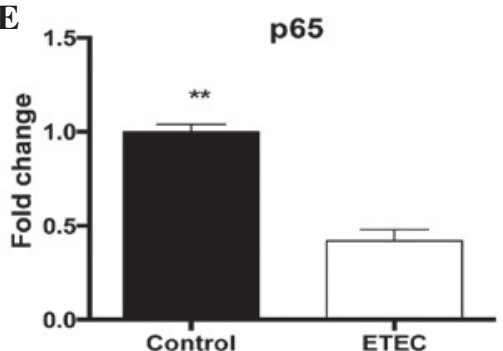

Figure 1. ETEC298 alters the TLR4-NF-кB pathway and intestinal morphology. (A) Hematoxylin and eosin staining of ETEC298-infected and PBS control groups (magnification, x100). (B) Immunoblotting of TLR4 subsequent to ETEC or PBS (control) infection for 24 h. (C) Quantification of relative TLR4 abundance from data presented in (B). (D) Immunoblotting of nuclear p65 subsequent to ETEC or PBS (control) infection for $24 \mathrm{~h}$. (E) Quantification of relative p65 abundance from data presented in (D). Data are presented as the mean + standard error, $n=6,{ }^{\prime \prime} \mathrm{P}<0.05,{ }^{* *} \mathrm{P}<0.01$ (Student's t-test). ETEC, enterotoxigenic Escherichia coli; TLR4, toll-like receptor 4; NF- $\mathrm{BB}$, nuclear factor $\mathrm{\kappa B}$; PBS, phosphate-buffered saline; PCNA, proliferating cell nuclear antigen.

treatment with PBS. Microscopic analyses demonstrated that the most remarkable alterations following ETEC298-infection were the loss of microvilli in the jejunum (Fig. 1A).

\section{ETEC298 infection increases TLR4 expression and inhibits} $N F-\kappa B$ signaling. Infection with ETEC298 significantly $(\mathrm{P}<0.05)$ increased TLR4 protein expression in the jejunum $24 \mathrm{~h}$ post-infection (Fig. 1B and C), in agreement with a previous study that demonstrated that ETEC infection significantly enhanced TLR4 gene expression in a piglet infection model (21). However, ETEC298 infection significantly $(\mathrm{P}<0.01)$ reduced $\mathrm{p} 65$ protein abundance in the nucleus (Fig. 1D and E), suggesting that ETEC infection inhibits the NF- $\mathrm{KB}$ signaling pathway.

ETEC298 infection activates the MAPK pathway. To determine the effect of ETEC on p38 MAPK, ERK1/2 and JNK activation, the activation-associated phosphorylation of these kinases was quantified using phosphorylation-specific antibodies. While ETEC infection was observed to have no significant effect on total p38, ERK1/2 and JNK protein levels, it induced significant phosphorylation of p38 $(\mathrm{P}<0.01)$ and ERK1/2 $(\mathrm{P}<0.05)$, without significant impact on JNK phosphorylation (Fig. 2).

ETEC infection promotes inflammation. Inflammatory responses are intended to disarm or destroy invading microorganisms, remove irritants and prepare for tissue repair or wound healing. The expression levels of the pro-inflammatory cytokines $I L-1 \beta, I L-6, I L-8$ and $T N F-\alpha$ following ETEC infection were measured. $I L-17 A$ expression was also measured due to the fact that it also serves a vital regulatory role in intestinal function. ETEC infection was observed to significantly $(\mathrm{P}<0.05)$ reduce the expression of all measured cytokines in the jejunum (Fig. 3).
ETEC affects intestinal immunity. Intestinal immune regulators serve an essential role in protection against bacterial invasion and maintenance of mucosal homeostasis (22-24). These regulators contain $\mathrm{J}$-chain, the polymeric immunoglobulin receptor (pIgR), mucin 2 and 4, $\alpha$-defensins, cryptdin-related sequence (CRS) peptides, lysozyme C, secretory group IIA phospholipase A2 (sPLA2), regenerating islet-derived $3 \gamma$ (REG3 $\gamma$ ) and RNase angiogenin 4 (ANG4) in mice $(15,25)$. As presented in Table I, ETEC298 infection significantly reduced the expression of $\mathrm{IIgR}, J$-chain and Lyz2 (lysozyme) $(\mathrm{P}<0.05)$, however significantly increased CRS4C (cysteine-rich sequence 4C) and CRSIC expression levels $(\mathrm{P}<0.05)$, and had little effect on other genes in the jejunum. In the ileum, the ETEC298 group had significantly reduced $(\mathrm{P}<0.05)$ expression of $p I g R$ and $C R S 1 C$, however increased $(\mathrm{P}<0.05)$ the expression of mucin 2, Lyz and cryptdin-5 compared with the control group, while no significant difference between the infected and control groups was observed for the remaining factors (Table II).

\section{Discussion}

In present study, ETEC infection was observed to reduce the expression levels of pro-inflammatory cytokines, including $I L-1 \beta, I L-6, I L-8, T N F-\alpha$ and $I L-17 A$, indicating ETEC infection represses the intestinal inflammatory responses in mice. However, Roselli et al (26) observed that ETEC (K88) infection promotes neutrophil transmigration, the expression of chemokines essential for neutrophil migration, such as IL-8, growth-related oncogene- $\alpha$ and epithelial neutrophil-activating peptide-78, and the expression of IL-1 $\beta$ and TNF- $\alpha$, which are regulators of chemokine expression, in Caco- 2 cells. In addition, similar results have been observed in intestinal epithelial IPI-2I cells (27), differentiated porcine intestinal epithelial IPEC-1 cells (28), IPEC J2 cells (29) and in vivo 


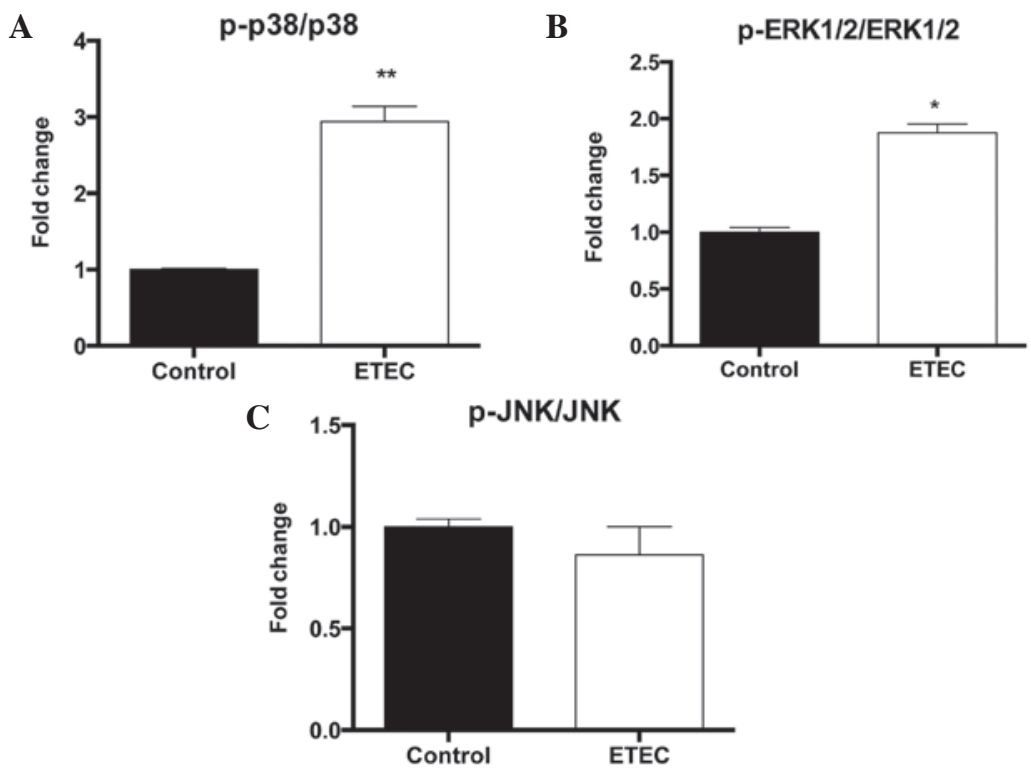

Figure 2. ETEC infection activates mitogen-activated protein kinases. (A) Quantification of the ratio of p-p38 to total p38 from immunoblotting data (B) Quantification of the ratio of p-ERK1/2 to total ERK1/2 from immunoblotting data. (C) Quantification of the ratio of p-JNK to total JNK from immunoblotting data. Data are presented as the mean + standard error, $n=6,{ }^{*} \mathrm{P}<0.05,{ }^{* *} \mathrm{P}<0.01$ (Student's t-test). ETEC, Escherichia coli; p-, phosphorylated; ERK, extracellular signal-related kinase; JNK, c-Jun N-terminal kinase.
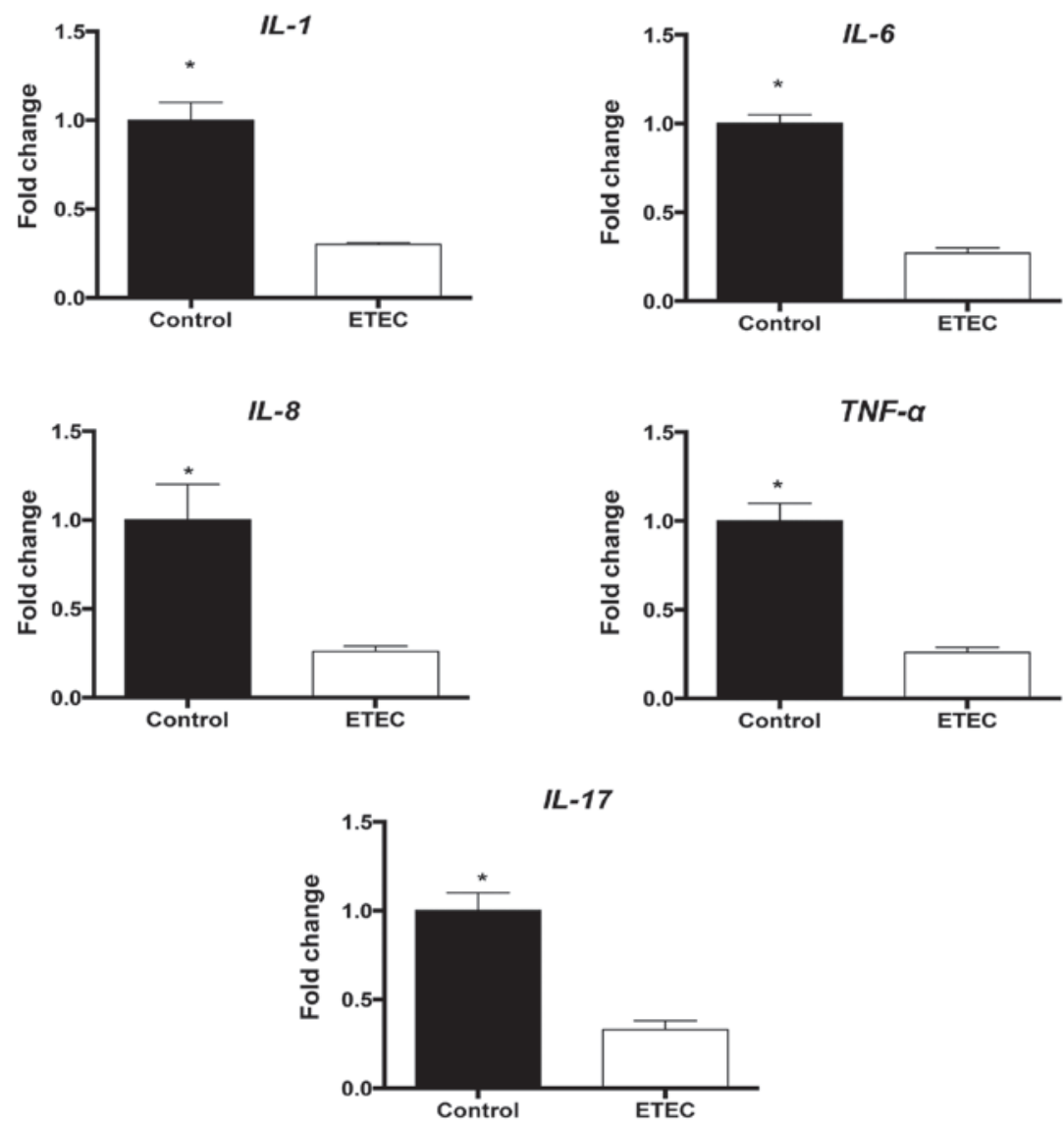

Figure 3. ETEC SEC470 infection inhibits pro-inflammatory cytokine expression. Pro-inflammatory cytokines (IL-1 $\beta,-6,-17$ and -8 and TNF- $\alpha$ ) gene expression in jejunum was detected using reverse transcription-quantitative polymerase chain reaction following ETEC298 or phosphate-buffered saline (control) infection for $24 \mathrm{~h}$. Data are presented as the mean + standard error, $n=6,{ }^{\prime} \mathrm{P}<0.05$ (Student's t-test). ETEC, Escherichia coli; IL, interleukin; TNF- $\alpha$; tumor necrosis factor $\alpha$.

with piglets (30). An additional study demonstrated that ETEC (K88) induces the secretion of IL-6 and IL-8 in intestinal epithelial cells associated with F4 fimbriae and flagellin (31). Furthermore, Ren et al (18) observed that ETEC infection 
Table I. Relative expression of intestinal innate immune factors in the jejunum in the ETEC298 and control groups.

\begin{tabular}{|c|c|c|}
\hline Factor & ETEC298 & Control group \\
\hline J-chain & $0.34 \pm 0.06^{\mathrm{a}}$ & $1.00 \pm 0.09$ \\
\hline$p I g R$ & $0.49 \pm 0.06^{\mathrm{a}}$ & $1.00 \pm 0.21$ \\
\hline Mucin 2 & $0.22 \pm 0.02$ & $1.00 \pm 0.15$ \\
\hline Mucin 4 & $2.68 \pm 1.46$ & $1.00 \pm 0.34$ \\
\hline Cryptdin-1 & $1.70 \pm 0.36$ & $1.00 \pm 0.12$ \\
\hline Cryptdin-4 & $0.98 \pm 0.29$ & $1.00 \pm 0.33$ \\
\hline Cryptdin-5 & $1.54 \pm 0.36$ & $1.00 \pm 0.12$ \\
\hline$C R S-1 C$ & $4.45 \pm 1.15^{\mathrm{a}}$ & $1.00 \pm 0.15$ \\
\hline$C R S-4 C$ & $3.84 \pm 0.83^{\mathrm{a}}$ & $1.00 \pm 0.12$ \\
\hline sPLA2 & $0.75 \pm 0.21$ & $1.00 \pm 0.16$ \\
\hline ANG4 & $1.10 \pm 0.23$ & $1.00 \pm 0.11$ \\
\hline$R E G 3 \gamma$ & $0.98 \pm 0.14$ & $1.00 \pm 0.09$ \\
\hline$L y z 2$ & $0.25 \pm 0.04^{\mathrm{a}}$ & $1.00 \pm 0.18$ \\
\hline
\end{tabular}

Mice were orally infected with ETEC298 or phosphate-buffered saline (control group). Values are presented as the mean \pm standard error, $\mathrm{n}=6$. ${ }^{\mathrm{a}} \mathrm{P}<0.05$, vs. the control group. $\mathrm{pIg} R$, polymeric immunoglobulin receptor; $C R S$, cryptdin-related sequence; $s P L A 2$, secretory group IIA phospholipase A2; ANG4, RNase angiogenin 4; REG3 $\gamma$, regenerating islet-derived $3 \gamma ;$ Lyz2, lysozyme 2 .

Table II. Relative expression of intestinal innate immune factors in the ileum in the ETEC298 and control groups.

\begin{tabular}{|c|c|c|}
\hline Factor & ETEC298 & Control group \\
\hline J-chain & $0.72 \pm 0.10$ & $1.00 \pm 0.10$ \\
\hline $\operatorname{pIg} R$ & $0.50 \pm 0.10^{\mathrm{a}}$ & $1.00 \pm 0.13$ \\
\hline Mucin 2 & $2.00 \pm 0.75^{\mathrm{a}}$ & $1.00 \pm 0.08$ \\
\hline Mucin 4 & $0.90 \pm 0.36$ & $1.00 \pm 0.27$ \\
\hline Cryptdin-1 & $1.10 \pm 0.14$ & $1.00 \pm 0.09$ \\
\hline Cryptdin-4 & $1.54 \pm 0.27$ & $1.00 \pm 0.26$ \\
\hline Cryptdin-5 & $1.85 \pm 0.30^{\mathrm{a}}$ & $1.00 \pm 0.11$ \\
\hline$C R S-1 C$ & $0.47 \pm 0.10^{\mathrm{a}}$ & $1.00 \pm 0.28$ \\
\hline$C R S-4 C$ & $1.26 \pm 0.21$ & $1.00 \pm 0.16$ \\
\hline sPLA2 & $0.91 \pm 0.17$ & $1.00 \pm 0.14$ \\
\hline ANG4 & $1.01 \pm 0.25$ & $1.00 \pm 0.17$ \\
\hline$R E G 3 \gamma$ & $0.25 \pm 0.11$ & $1.00 \pm 0.79$ \\
\hline$L y z 2$ & $1.69 \pm 0.14^{\mathrm{a}}$ & $1.00 \pm 0.21$ \\
\hline
\end{tabular}

Mice were orally infected with ETEC298 or phosphate-buffered saline (control group). Values are presented as the mean \pm standard error, $\mathrm{n}=6$. ${ }^{\mathrm{a}} \mathrm{P}<0.05$, vs. the control group. $\mathrm{pIg} R$, polymeric immunoglobulin receptor; $C R S$, cryptdin-related sequence; $S P L A 2$, secretory group IIA phospholipase A2; ANG4, RNase angiogenin $4 ; R E G 3 \gamma$, regenerating islet-derived $3 \gamma ; L y z 2$, lysozyme 2 .

promotes the expression of pro-inflammatory cytokines through the activation of the NF- $\kappa \mathrm{B}$ and MAPK pathways. A possible explanation for these discrepancies may be the fact that different infectious models and bacterial strains were used. The strain used in the current study lacks LT, unlike the ETEC strain previously used (18). Other clinical isolated strains, which lack LT, have additionally been observed to fail to promote the expression of pro-inflammatory cytokines in mouse jejunum subsequent to $24 \mathrm{~h}$ infection (Liu et al, unpublished data).

The inhibition of the expression of pro-inflammatory cytokines may be via the inactivation of the $\mathrm{NF}-\kappa \mathrm{B}$ pathway subsequent to ETEC infection. In line with the results of the current study, Wang and Hardwidge (7) reported that ETEC blocks $N F-\kappa B$ signaling by secreting a heat-stable, proteinaceous factor. The ETEC supernatant has been observed to significantly block the degradation of the NF- $\kappa \mathrm{B}$ inhibitor I $\kappa \mathrm{B} \alpha$ by preventing I $\kappa \mathrm{B} \alpha$ polyubiquitination, without affecting $\mathrm{I} \kappa \mathrm{B} \alpha$ phosphorylation (7). However, numerous studies have suggested that ETEC infection may promote the activation of the $\mathrm{NF}-\kappa \mathrm{B}$ pathway in intestinal epithelial cells (HCT-8 cells) (32) and porcine intestinal epitheliocyte (PIE) cells (33). Thus, it is notable to investigate the underling reason for this discrepancy. In the current study, ETEC infection was observed to activate the MAPK pathway via the increased phosphorylation of $\mathrm{p} 38$ and ERK1/2. In agreement with the results of the current study, ETEC was observed to be able to activate MAPK signaling pathway in intestinal epithelial cells (HCT-8 cells) through mechanisms that are primarily dependent upon LT (32). Shimazu et al (33) also demonstrated that ETEC infection promotes MAPK activation in PIE cells. A possible explanation for MAPK activation following ETEC infection is the activation of TLR signaling. It has been widely reported that ETEC infection activates TLR signaling in PIE cells (33), human intestinal Caco-2/TC7 cells and intestinal explants isolated from 5-week-old crossbreed Pietrain/Duroc/Large-White piglets (4), and weaned piglets (34).

ETEC infection affects intestinal innate immunity, including secretory immunoglobulin A $(\operatorname{sig} \mathrm{A}$, based on the mRNA expression of J-chain and pIgR), goblet cells (based on the mRNA expression of Mucin 2) and Paneth cells (based on the mRNA expression of CRS-1C, CRS-4C, Lyz2 and Cryptdin-5). Numerous studies have indicated that ETEC infection affects the secretion of $\operatorname{sIgA}$ in piglets $(34,35)$. However, little literatures have reported the effect of ETEC infection on the intestinal goblet and Paneth cell function in vivo. Considering that ETEC infection affects the expression of antibacterial substances expressed by goblet and Paneth cell, thus it is interesting to explore the effect of ETEC infection on Paneth cell and goblet cell.

In conclusion, ETEC infection inhibits the expression in mouse jejunum of pro-inflammatory cytokines associated with the inhibition of $\mathrm{NF}-\kappa \mathrm{B}$. ETEC infection promotes the activation of the MAPK pathway in the mouse model. Furthermore, ETEC infection affects the intestinal sIgA levels, and the function of goblet and Paneth cells.

\section{References}

1. Field M: Intestinal ion transport and the pathophysiology of diarrhea. J Clin Invest 111: 931-943, 2003.

2. Moeser AJ and Blikslager AT: Mechanisms of porcine diarrheal disease. J Am Vet Med Assoc 231: 56-67, 2007.

3. Tang Y, Li F, Tan B, Liu G, Kong X, Hardwidge PR and Yin Y: Enterotoxigenic Escherichia coli infection induces intestinal epithelial cell autophagy. Vet Microbiol 171: 160-164, 2014. 
4. Finamore A, Roselli M, Imbinto A, Seeboth J, Oswald IP and Mengheri E: Lactobacillus amylovorus inhibits the TLR4 inflammatory signaling triggered by enterotoxigenic Escherichia coli via modulation of the negative regulators and involvement of TLR 2 in intestinal Caco-2 cells and pig explants. PLoS One 9: e94891, 2014.

5. Zhu YH, Li XQ, Zhang W, Zhou D, Liu HY and Wang JF: Dose-dependent effects of Lactobacillus rhamnosus on serum interleukin-17 production and intestinal T-cell responses in pigs challenged with Escherichia coli. Appl Environ Microbiol 80 1787-1798, 2014.

6. McLamb BL, Gibson AJ, Overman EL, Stahl C and Moeser AJ: Early weaning stress in pigs impairs innate mucosal immune responses to enterotoxigenic $E$. coli challenge and exacerbates intestinal injury and clinical disease. PLoS One 8: e59838, 2013

7. Wang X and Hardwidge PR: Enterotoxigenic Escherichia coli prevents host $\mathrm{NF}-\kappa \mathrm{B}$ activation by targeting $\mathrm{I} \kappa \mathrm{B} \alpha$ polyubiquitination. Infect Immun 80: 4417-4425, 2012.

8. Ghosal A, Chatterjee NS, Chou T and Said HM: Enterotoxigenic Escherichia coli infection and intestinal thiamin uptake: Studies with intestinal epithelial Caco-2 monolayers. Am J Physiol Cell Physiol 305: C1185-C1191, 2013.

9. Nakashima R, Kamata $\mathrm{Y}$ and Nishikawa Y: Effects of Escherichia coli heat-s enterotoxin and guanylin on the barrier integrity of intestinal epithelial T84 cells. Vet Immunol Immunopathol 152: 78-81, 2013.

10. Johnson AM, Kaushik RS and Hardwidge PR: Disruption of transepithelial resistance by enterotoxigenic Escherichia coli. Vet Microbiol 141: 115-119, 2010.

11. Egberts HJ, de Groot EC, van Dijk JE, Vellenga L and Mouwen JM: Tight junctional structure and permeability of porcine jejunum after enterotoxic Escherichia coli infection. Res Vet Sci 55: 10-14, 1993

12. Syed HC and Dubreuil JD: Escherichia coli STb toxin induces apoptosis in intestinal epithelial cell lines. Microb Pathog 53 147-153, 2012.

13. Johnson AM, Kaushik RS, Rotella NJ and Hardwidge PR Enterotoxigenic Escherichia coli modulates host intestinal cel membrane asymmetry and metabolic activity. Infect Immun 77: 341-347, 2009

14. Ren WK, Yin J, Zhu XP, Liu G, Li NZ, Peng YY and Yin YL: Glutamine on intestinal inflammation: A mechanistic perspective. Eur J Inflamm 11: 315-326, 2013.

15. Ren W, Chen S, Yin J, Duan J, Li T, Liu G, Feng Z, Tan B, Yin Y and Wu G: Dietary arginine supplementation of mice alters the microbial population and activates intestinal innate immunity. J Nutr 144: 988-995, 2014.

16. Chen X, Huan H, Wan T, Wang L, Gao S and Jiao X: Antigenic determinants analysis and detection of virulence factors in F18 fimbriae Escherichia coli strains isolated from pigs. Wei Sheng Wu Xue Bao 54: 236-242, 2014 (In Chinese).

17. Allen KP, Randolph MM and Fleckenstein JM: Importance of heat-labile enterotoxin in colonization of the adult mouse smal intestine by human enterotoxigenic Escherichia coli strains. Infect Immun 74: 869-875, 2006

18. Ren W, Yin J, Duan J, Liu G, Zhu X, Chen S, Li T, Wang S, Tang Y and Hardwidge PR: Mouse jejunum innate immune responses altered by enterotoxigenic Escherichia coli (ETEC) infection. Microbes Infect 16: 954-961, 2014

19. Ren WK, Liu SP, Chen S, Zhang F, Li N, Yin J, Peng Y, Wu L, Liu G, Yin Y and Wu G: Dietary L-glutamine supplementation increases Pasteurella multocida burden and the expression of its major virulence factors in mice. Amino Acids 45: 947-955, 2013.

20. Ren W, Luo W, Wu M, Liu G, Yu X, Fang J, Li T, Yin Y and Wu G: Dietary L-glutamine supplementation improves pregnancy outcome in mice infected with type- 2 porcine circovirus. Amino Acids 45: 479-488, 2013.
21. Hermes RG, Manzanilla EG, Martín-Orúe SM, Pérez JF and Klasing KC: Influence of dietary ingredients on in vitro inflammatory response of intestinal porcine epithelial cells challenged by an enterotoxigenic Escherichia coli (K88). Comp Immunol Microbiol Infect Dis 34: 479-488, 2011.

22. Pabst O: New concepts in the generation and functions of $\operatorname{IgA}$. Nat Rev Immunol 12: 821-832, 2012.

23. Vereecke L, Beyaert R and van Loo G: Enterocyte death and intestinal barrier maintenance in homeostasis and disease. Trends Mol Med 17: 584-593, 2011.

24. Bevins CL and Salzman NH: Paneth cells, antimicrobial peptides and maintenance of intestinal homeostasis. Nat Rev Microbiol 9: 356-368, 2011.

25. Ren W, Duan J, Yin J, Liu G, Cao Z, Xiong X, Chen S, Li T, Yin Y, Hou Y and Wu G: Dietary L-glutamine supplementation modulates microbial community and activates innate immunity in the mouse intestine. Amino Acids 46: 2403-2413, 2014.

26. Roselli M, Finamore A, Britti MS and Mengheri E: Probiotic bacteria Bifidobacterium animalis MB5 and Lactobacillus rhamnosus GG protect intestinal Caco-2 cells from the inflammation-associated response induced by enterotoxigenic Escherichia coli K88. Br J Nutr 95: 1177-1184, 2006.

27. Zanello G, Meurens F, Berri M, Chevaleyre C, Melo S, Auclair E and Salmon H: Saccharomyces cerevisiae decreases inflammatory responses induced by $\mathrm{F} 4+$ enterotoxigenic Escherichia coli in porcine intestinal epithelial cells. Vet Immunol Immunopathol 141: 133-138, 2011.

28. Zanello G, Berri M, Dupont J, Sizaret PY, D'Inca R, Salmon H and Meurens F: Saccharomyces cerevisiae modulates immune gene expressions and inhibits ETEC-mediated ERK1/2 and p38 signaling pathways in intestinal epithelial cells. PLoS One 6: e18573, 2011

29. Sargeant HR, Miller HM and Shaw MA: Inflammatory response of porcine epithelial IPEC J2 cells to enterotoxigenic $E$. coli infection is modulated by zinc supplementation. Mol Immunol 48: 2113-2121, 2011.

30. Sargeant HR, McDowall KJ, Miller HM and Shaw MA: Dietary zinc oxide affects the expression of genes associated with inflammation: Transcriptome analysis in piglets challenged with ETEC K88. Vet Immunol Immunopathol 137: 120-129, 2010.

31. Devriendt B, Stuyven E, Verdonck F, Goddeeris BM and Cox E: Enterotoxigenic Escherichia coli (K88) induce proinflammatory responses in porcine intestinal epithelial cells. Dev Comp Immunol 34: 1175-1182, 2010.

32. Wang X, Gao $X$ and Hardwidge PR: Heat-labile enterotoxin-induced activation of $N F-\kappa B$ and MAPK pathways in intestinal epithelial cells impacts enterotoxigenic Escherichia coli (ETEC) adherence. Cell Microbiol 14: 1231-1241, 2012

33. Shimazu T, Villena J, Tohno M, Fujie H, Hosoya S, Shimosato T, Aso H, Suda Y, Kawai Y, Saito T, et al: Immunobiotic Lactobacillus ensenii elicits anti-inflammatory activity in porcine intestinal epithelial cells by modulating negative regulators of the Toll-like receptor signaling pathway. Infect Immun 80: 276-288, 2012.

34. Xiao D, Tang Z, Yin Y, Zhang B, Hu X, Feng Z and Wang J: Effects of dietary administering chitosan on growth performance, jejunal morphology, jejunal mucosal sIgA, occluding, claudin-1 and TLR4 expression in weaned piglets challenged by enterotoxigenic Escherichia coli. Int Immunopharmacol 17: 670-676, 2013

35. Gao Y, Han F, Huang X, Rong Y, Yi H and Wang Y: Changes in gut microbial populations, intestinal morphology, expression of tight junction proteins and cytokine production between two pig breeds after challenge with Escherichia coli K88: A comparative study. J Anim Sci 91: 5614-5625, 2013. 